\title{
INVESTIGATION INTO THE POTENTIAL OF SINGLE PHOTON AIRBORNE LASER SCANNING TECHNOLOGY
}

\author{
C. Bernard ${ }^{1}$, J. P. Mills ${ }^{2, *}$, J. Talaya ${ }^{3}$, F. Remondino ${ }^{4}$ \\ ${ }^{1}$ Ecole Nationale des Sciences Géographiques (ENSG), Paris, France - charly.bernard@ensg.eu \\ ${ }^{2}$ School of Engineering, Newcastle University, Newcastle upon Tyne, UK - jon.mills@ newcastle.ac.uk \\ ${ }^{3}$ Institut Cartogràfic i Geològic de Catalunya (ICGC), Barcelona, Spain - julia.talaya@ @icgc.cat \\ ${ }^{4}$ 3D Optical Metrology (3DOM) unit, Bruno Kessler Foundation (FBK), Trento, Italy - remondino@ fbk.eu
}

Commission I, WG I/2

KEY WORDS: Airborne Laser Scanning, Geiger-Mode, Lidar, Mapping, Sensors, Single Photon

\begin{abstract}
:
Following several years of incremental technological developments in airborne laser scanning, the field is currently witnessing potentially revolutionary change through the introduction of new technologies, namely Single Photon (SP) and Geiger-Mode (GM) lidar. These new sensors potentially provide more efficient approaches to perform high-resolution 3D mapping, but seemingly to the detriment of accuracy and noise. Despite concerns, the technology is perceived to offer higher efficiency with respect to conventional airborne laser scanning and additional 3D mapping capability in terms of swath width, spatial resolution, acquisition time and density of range returns. These developments have led to some European National Mapping and Cadastral Agencies (NMCAs) to consider a potential change in their production pipelines, while some mapping companies are contemplating upgrading their existing airborne sensor systems. However, few open datasets and experiences are available to inform such decisions, and the community is keen to learn more about the technology and its deployment for 3D territorial mapping. These facts and considerations motivated EuroSDR Commission 1 to launch a new initiative in 2018 to better understand, explore and report to its NMCA membership the developments in SP and GM technologies. This paper describes the activities that have thus far been conducted as part of this EuroSDR initiative, comprising: a global on-line questionnaire into awareness of the technology; a preliminary investigation into a Leica SPL100 dataset of Easton, USA; and a community workshop at which a proposal to run an international benchmarking exercise was discussed.
\end{abstract}

\section{INTRODUCTION}

\subsection{Background}

Emergent technologies in the form of Single Photon (SP) and Geiger-Mode (GM) lidar are potentially on the cusp of revolutionising large area surveying and mapping. With respect to conventional lidar, SP and GM technology is generally perceived to offer higher efficiency in data acquisition, providing advanced $3 \mathrm{D}$ mapping capability in terms of swath width, spatial resolution, acquisition time and density of range returns. Simultaneously, the ability of such sensors to perform enhanced 3D mapping over conventional lidar are seemingly hampered by issues relating to lower point accuracy, single or fewer returns per pulse, and higher noise levels in the resultant datasets. Unfortunately, at present few open datasets and experiences are available to reliably inform users and practitioners about the advantages and disadvantages of this new technology, and the community is keen to learn more about its potential for deployment in 3D mapping applications. The research reported in this paper begins to address this need by (a) establishing the community's current perceptions regarding SP and GM lidar via garnered questionnaire responses, and (b) testing the validity of these perceptions through analysis of a typical SP lidar dataset. Moreover, the paper reports on a recent workshop dedicated to the topic of SP and GM lidar which brought together the international mapping community to discuss and deliberate the issues at hand. As a result of the reported activity, suggestions are made as to the next applied research steps that are required in order for the international community to reliably adopt these potentially revolutionary approaches to 3D mapping.

\subsection{SP and GM lidar technologies}

SP and GM lidar have emerged as exciting new developments in the airborne laser scanning sector. SP, through the Leica SPL100 sensor (Hexagon, 2019a) and GM, via Harris' proprietary collection program (Harris Geospatial Solutions, 2019) have generated increasing levels of interest amongst data providers, National Mapping and Cadastral Agencies (NMCAs) and other users world-wide. The technology uses single photon avalanche diode arrays (Zappa et al., 2007) to count and timetag single photons of energy returned from the back scattered signal. Harris' GM lidar utilises a $32 \times 128$ array to sample the full divergent backscattered beam (infrared, wavelength $1024 \mathrm{~nm})$ at the receiving optics. In contrast, Leica's SP lidar solution splits the initial green laser beam (wavelength $532 \mathrm{~nm}$ ) into $10 \times 10$ highly collimated beamlets and adopts a complementary $10 \times 10$ diode array to detect and measure the return signals. In both approaches, the higher sensitivity of the detector means that the instruments can be operated at higher flying heights, with associated benefits for survey efficiency. Unfortunately the sensors' characteristics also has implications

\footnotetext{
* Corresponding author
} 
on individual point precision / accuracy and for the undesired detection (at least in topographic mapping lidar) of aerosol particles and solar interference in the atmosphere. Moreover, single photon diode arrays are also susceptible to electronic effects such as crosstalk which can add to prevalent noise.

Degnan (2016) charts the development of SP lidar and compares the technology with its GM counterpart. Stoker et al. (2016) evaluated both SP and GM lidar for use in the United States 3D Elevation Program (3DEP). Whilst the technology was found not to meet the required specification for the 3DEP, the authors recognised that this was at least partly due to the specification having been written specifically for conventional lidar systems. Some of the sensor shortcomings identified in the study were reported as being addressed in later versions.

Recent reports on the use of such technology show promise for potential future application in fields such as forestry (Wästlund, 2018) and large area mapping of water surface heights (Mandlburger and Jutzi, 2018). A comparison between SP lidar and conventional full waveform lidar over the city of Vienna, Austria, was recently presented by Mandlburger and Lehner (2019).

\subsection{EuroSDR}

EuroSDR is a not-for-profit organisation linking NMCAs with research institutes and universities across Europe for the purpose of undertaking applied research in spatial data provision, management and delivery. The various EuroSDR Commissions conduct applied research programmes into topics of real-world relevance to its membership, as determined by the organisation's Board of Delegates. EuroSDR Commission 1 focusses on primary data acquisition, its mission being to explore, test and validate platforms, sensors and algorithms to acquire geospatial data, with emphasis on accuracy, reliability and standardization of data processing procedures.

With interest in SP and GM lidar growing, but few open datasets and critical experiences available to inform decisions on its adoption (particularly in Europe), EuroSDR Commission 1 decided to launch a new initiative in 2018 to better understand, explore and report the developments in these technologies. This paper describes the activities that have been conducted thus far as part of this EuroSDR initiative. Section 2 reports responses made to an online questionnaire conducted to help gauge the current status and perceptions of SP and GM lidar technologies across the mapping community. Section 3 provides preliminary analysis of a typical SP lidar dataset. Section 4 updates the community on recent, ongoing and future actions and Section 5 summarises the position to date.

\section{GLOBAL AWARENESS OF SP / GM LIDAR}

\subsection{On-line questionnaire}

EuroSDR Commission 1 ran an on-line questionnaire to inform its future activities over the period August to September 2018. The questionnaire included a substantive section on the potential of single photon technologies, with respondents asked to detail their understanding and experiences. The questionnaire was distributed via EuroSDR and ISPRS networks, and garnered 120 responses from 42 different countries across six continents (Figure 1). Responses were made from one equipment manufacturer ( $1 \%$ of responses), eight geospatial data providers (7\%), 20 NMCAs $(17 \%), 83$ University or research institutes (69 \%), eight "other" (mainly different government departments, $7 \%$ ). Taken as a whole, and considering occasional multiple responses from single institutions, more than 100 different organisations were represented.

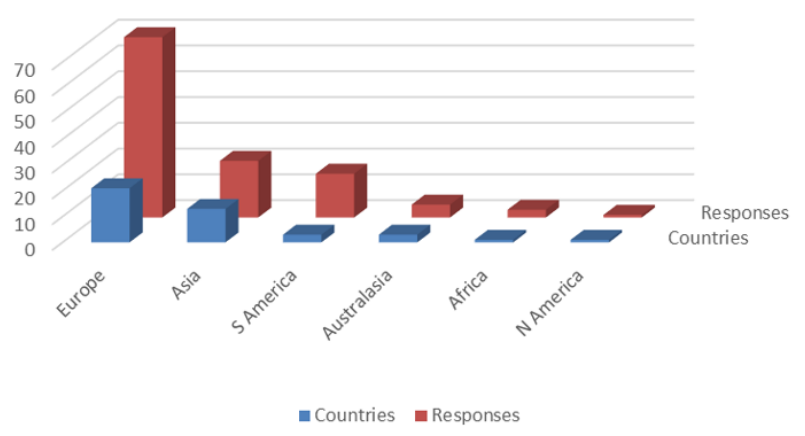

Figure 1. Summary of questionnaire responses by continent.

\subsection{Awareness}

Of the 120 responses received, 51 (43\%) respondents claimed to be aware of SP/GM lidar. This figure rose to $70 \%$ when only NMCA responses were considered (14 of 20 replies). Of the 51 respondents that were aware of the technology, only $12(24 \%)$ had any experience whatsoever in its acquisition or application, with the figure rising to $43 \%$ amongst NMCA replies.

\subsection{Perspectives}

The 51 respondents who claimed to be aware of SP and GM lidar were asked further questions related to, for example, the perceived applications of the technology and which characteristics of systems were deemed to be most important for their area of specialism, with analysis broken down into different classes of respondent.

2.3.1 Perceived advantages and disadvantages: various advantages and disadvantages of the technology were listed in free text responses, but responses often admitted to being confused and / or lacking in absolute clarity. Common advantages that were listed included: higher point density; larger areal coverage; high altitude acquisition; faster survey times. Less commonly referenced advantages included the potential ability for SP/GM lidar to penetrate vegetation and water (SP lidar), as well as less dependency on prevalent atmospheric conditions. The dominant answers in terms of disadvantages related to higher levels of noise; lower accuracy point measurements; economic cost of investment. Other perceived negatives related to the lack of substantial independent analysis; concerns over vegetation penetration capability; data volumes and processing software.

2.3.2 Potential applications: respondents saw many potential applications for the technology, the responses perhaps best summarised by the statement, "basically all large-area applications where detection of object is not based on few points". A number of responses commented along the lines of "we need more information about this technology before deciding what it will be useful for". The most popular applications perceived amongst NMCA respondents are listed in Figure 2. 


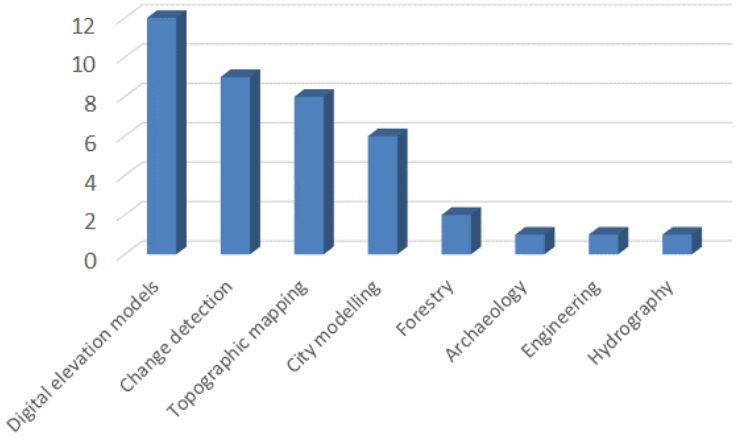

Figure 2. Potential applications of SP/GM lidar as perceived by 20 NMCA respondents.

2.3.3 Important characteristics: respondents were asked to rate the importance of various characteristics on a four-point scale, from unimportant to very important. Total weighted responses (out of 100) for each characteristic are given in Figure 3. Height point accuracy, spatial point density, cost, vegetation penetration and planimetric point accuracy were rated as the most important, with little to choose between them.

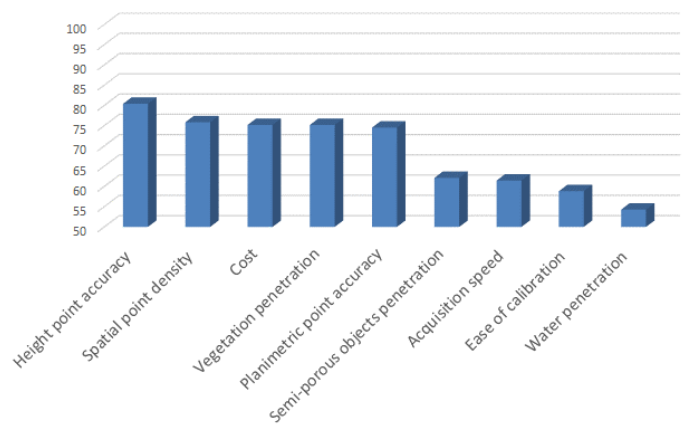

Figure 3. Important characteristics of SP/GM lidar.

NMCA responses were interesting, most notably for rating height point accuracy behind planimetric point accuracy and spatial point density (Figure 4). This perhaps reflects the primary requirement for NMCAs in terms of topographic mapping and the continued importance of planimetry over height. In free text responses, respondents also highlighted the need for consistent data quality over large areas, automation in data processing, usefulness of intensity reading and the relation to target properties (reflectance), suitable platforms (including unmanned aerial system capability), as well as accessibility.

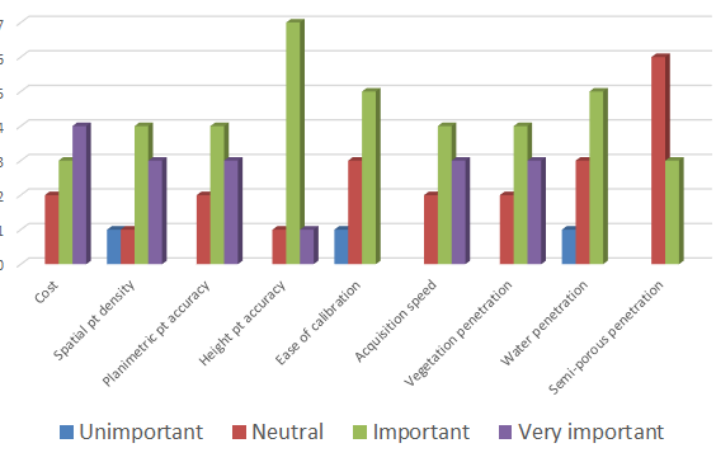

Figure 4. Important characteristics of SP/GM lidar as perceived by 20 NMCA respondents.
2.3.4 Requisite accuracies: the responses relating to the accuracy requirement for measuring various infrastructure are reported in Figure 5a for NMCAs and $5 \mathrm{~b}$ for all other responses. Interestingly, NMCA needs / expectations vary slightly from the other responses, most notably in buildings where a $\mathrm{cm}$ level accuracy was specified by the majority of respondents. Clearly, providing such accuracy from any airborne remote sensing mapping technology is a significant challenge.

(a) NMCA responses

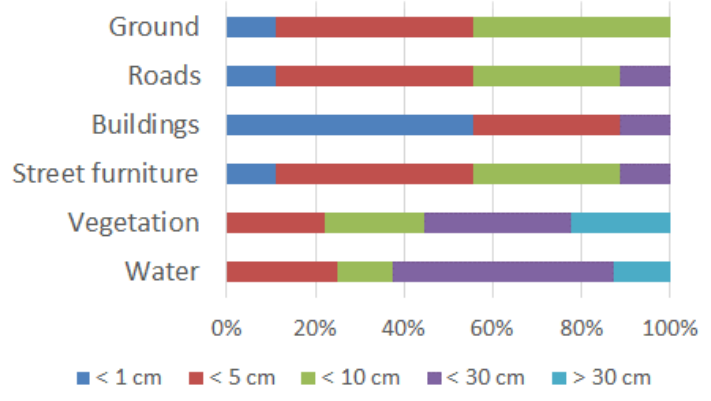

(b) Other responses

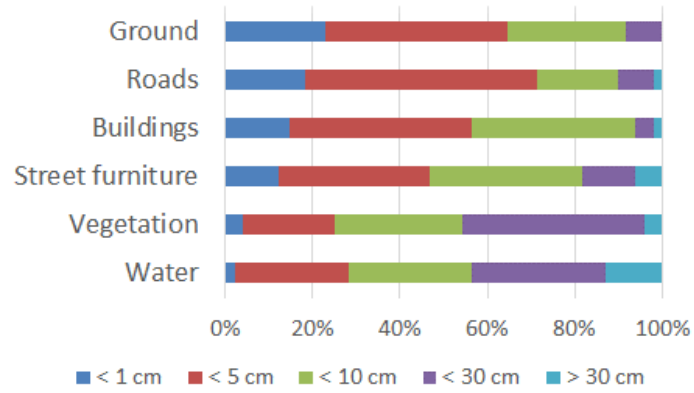

Figure 5. Requisite accuracy for various infrastructure: (a) NMCA responses; (b) all other responses.

2.3.5 Additional observations: the questionnaire concluded by asking respondents for any additional observations they wished to make about the technology. 24 additional comments were recorded, many observing the need for new investigations and benchmarking to provide independent quality statements. Other observations included the requirement for evaluation of sensor performance over snow and ice; practical considerations such as when the switchover from conventional lidar becomes cost effective; effectiveness of existing algorithms and workflows; potential georeferencing (strip matching) issues.

\subsection{Questionnaire summary}

In summary, all respondents were positive in their enthusiasm to see EuroSDR conduct further independent investigations into the area of SP and GM lidar technology. Reflecting this, there was also a positive response from a majority of respondents when asked whether they would participate in future EuroSDR activity relating to workshops and benchmarking exercises on the topic of SP and GM lidar.

\section{PRELIMINARY ASSESSMENT OF SP LIDAR}

\subsection{Background}

In parallel to the questionnaire reported in Section 2, EuroSDR conducted a preliminary investigation into the potential of SPL 
during the summer of 2018. A 2017 dataset acquired using a Leica SPL100 (Hexagon, 2019) over Easton, USA, was analysed. The data was kindly provided by Hexagon Geosystems for the purposes of the study. Unfortunately, with no ground truth information available, rigorous analysis into the accuracy of the dataset was not feasible and so figures reported herein instead focus only on the precision and various characteristics of the dataset.

\subsection{Test dataset and software}

Nominal parameters for the provided test dataset are presented in Table 1. The overflown area of Easton is located near Washington D.C., Maryland, USA. The dataset consists of heterogeneous land cover, including urban areas (Easton town), water bodies (bays and rivers), woodland (leaf off, given the acquisition date of 25 January 2017), etc., although elevation change across the area is minimal. The dataset consists of three strips, each of length $20 \mathrm{~km}$ with lateral strip overlaps of approximately $60 \%$ (Figure 6).

\begin{tabular}{|l|l|}
\hline Parameter & Value \\
\hline Date & 25 January 2017 \\
Time of day & Afternoon \\
Location & Easton, MD, USA \\
Flying height & 12,300 feet $($ c. $3750 \mathrm{~m})$ \\
Flying speed & 200 knots $($ c. $370 \mathrm{~km} / \mathrm{h})$ \\
Scan pattern & Conical \\
Conical scan angle to nadir & 15 degrees \\
Lateral overlap & $60 \%$ \\
Number of flight lines & 3 \\
\hline
\end{tabular}

Table 1. Data acquisition parameters for Easton dataset (courtesy Hexagon Geosystems).

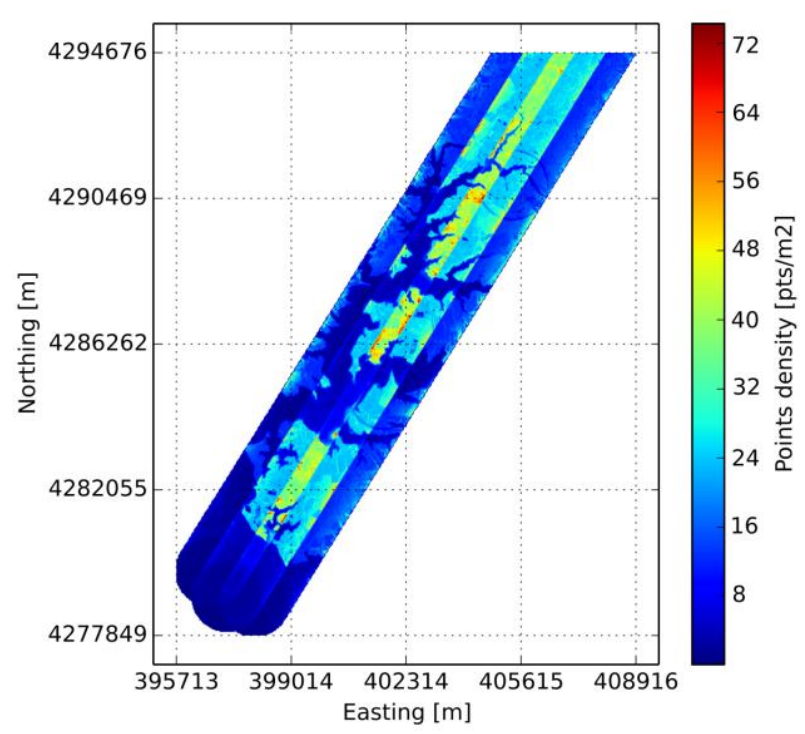

Figure 6. Extent of Easton dataset flight lines (note, data has been sub-sampled to enable visualisation)

Each individual flight line, provided in LAS format, consisted of c. 350 million points, meaning the total base dataset contained in excess of 1 billion data points with a combined file size greater than $6 \mathrm{~GB}$. To ease processing, the study area was therefore subdivided into $1 \mathrm{x} 1 \mathrm{~km}$ tiles (Figure 7) using Terrasolid TerraScan software, which was also utilised to perform a slope-based ground classification. Further analysis was conducted using Python, making use of the laspy library to read the provided LAS files, and the open source software CloudCompare was also utilised to visualise the datasets.

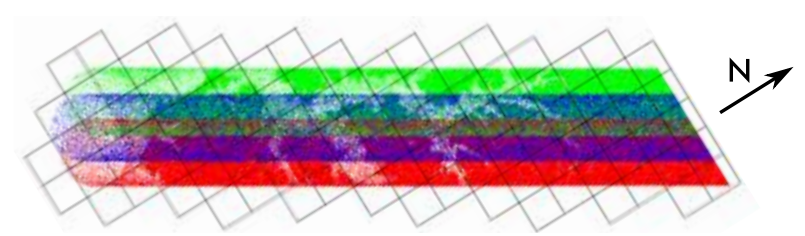

Figure 7. 1 x $1 \mathrm{~km}$ tiling of Easton dataset flight lines.

Visual inspection of the dataset showed that there were very few "flying" points evident, suggesting the dataset had been preprocessed prior to delivery, but it is unknown as to exactly what level of pre-processing had been performed. The Leica SPL100 sensor is capable of recording multiple returns, with up to 10 returns per channel reported. However, analysis of the dataset, in which every $100^{\text {th }}$ point was sampled, revealed that less than $4 \%$ of the points observed by the sensor recorded more than a single echo (Table 2). No return recorded more than four echoes. The fact that the data was captured under leaf-off conditions may have some influence on this result, as could suspected pre-processing to remove "flying" points.

\begin{tabular}{|l|r|r|}
\hline No. of returns & No. of points & \% of total points \\
\hline 1 & $10,410,935$ & 96.016 \\
2 & 420,912 & 3.882 \\
3 & 10,986 & 0.101 \\
4 & 72 & 0.001 \\
\hline
\end{tabular}

Table 2. Analysis of Leica SPL100 multiple returns for Easton dataset (1\% sample).

An interesting artefact that occurred at two instances in one of the three scan lines resulted from the apparent momentary drop out in recording lidar returns (Figure 8).

(a)

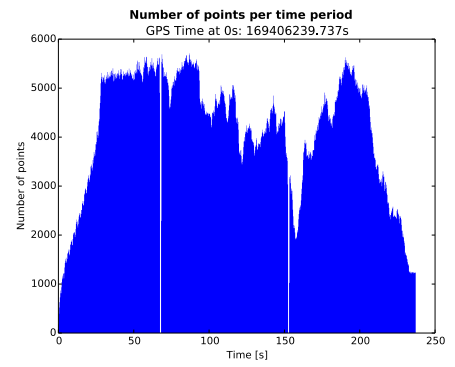

(b)

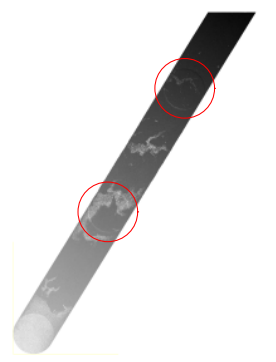

(c)

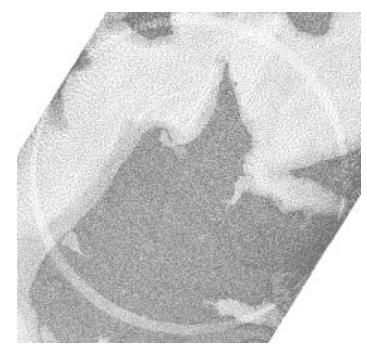

Figure 8. Circular artefacts: (a) apparent momentary dropouts in Leica SPL100 data recording; (b) resultant artefacts in one swath; (c) close-up of one artefact showing resultant impact on point density. 
Figure 8a shows the number of points collected per time period for this strip, where two gaps in data recording are clearly visible. This manifests itself in the point cloud as areas of lower point density, with the circular recording pattern of the sensor clearly visible in the point cloud (Figures $8 \mathrm{~b}$ and $8 \mathrm{c}$ ). This observation does highlight one advantage of the circular scan pattern adopted by the Leica SPL100: the scanner covers the full swath width at least twice per flight line with a fore and aft look. Hence, if the scanner has an issue which disables it momentarily, as appears to be the case here, the affected area will nevertheless be covered at least once by the instrument.

\subsection{Point density and distribution}

3.3.1 Simulation: numerous flight parameters contribute to the final point density and distribution in any lidar survey. In order to consider the influence of these, a simulator was developed to create synthetic data to analyse the theoretical coverage and point density of a Leica SPL100 survey. Figure 9 shows the parameters considered in the developed simulator to determine the resultant position of a $10 \times 10$ matrix of simulated Leica SPL100 ground points, M, which were:

- Flying speed, v;

- Flying height, h;

- Pulse repetition frequency, f;

- Conical scan angle to nadir, $\theta$;

- Circular scan angle and its angular speed, $\varphi$.
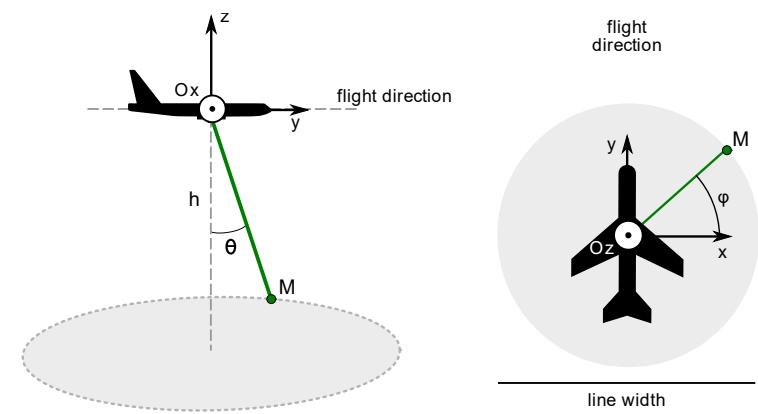

Figure 9. Schematic showing lidar simulator parameters.

Parameters were chosen to match the details of the Easton survey (Table 1), with the exception of angular speed, for which no data was provided, and the value was therefore estimated at 200 rotations per second. Figure 10 shows the resultant theoretical point densities for a single strip of data acquired over a $50 \mathrm{~s}$ period in both planimetry and height.

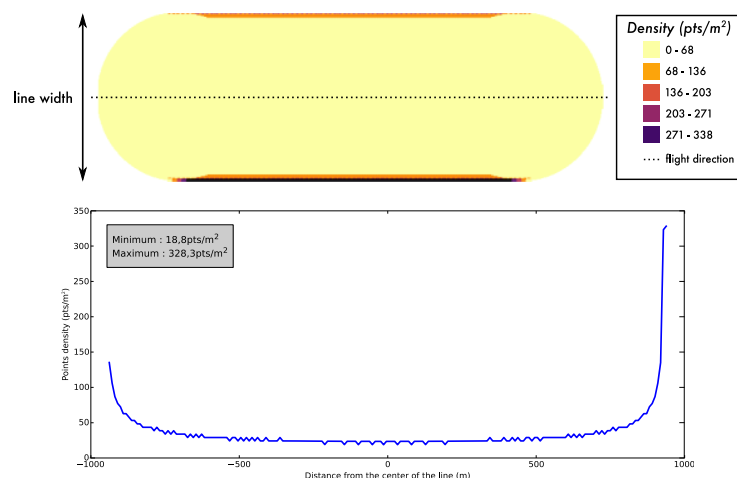

Figure 10. Theoretical Leica SPL100 point density map (top) and swath cross-section (bottom).
Examination of Figure 10 reveals that the conical scan mechanism used in the Leica SPL100 results in largely homogenous density for much of the swath width, but that density increases significantly at the lateral edge of a strip. This phenomenon is clearly also evident when examining samples of the Easton dataset (e.g. Figure 11).

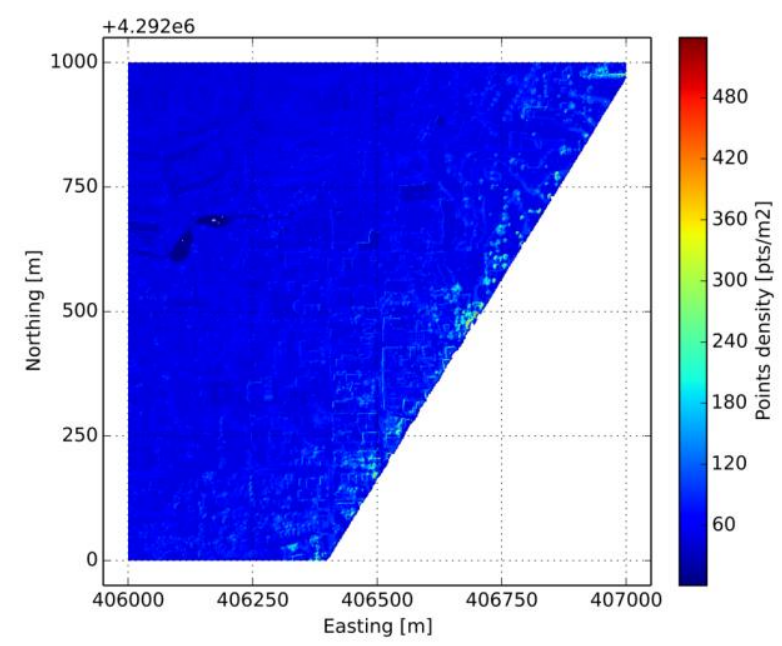

Figure 11. Sample Easton $1 \mathrm{x} 1 \mathrm{~km}$ tile, showing a single flight line. Note increased point density at periphery of swath.

3.3.2 Simulation: Easton dataset assessment: eight $1 \times 1 \mathrm{~km}$ tiles were selected to determine achieved point density in the Easton dataset. Only individual flight lines were analysed, to avoid over-estimating the point density on the basis of measuring in overlapping strip areas. A Python script calculated point density for the eight sampled point clouds. This was achieved for different land cover types by dividing each $1 \mathrm{~km}^{2}$ dataset into $1000 \times 1000$ sub-tiles and using "ground truth" from an orthophoto to classify the $1 \mathrm{~m}^{2}$ sub-tiles. The resulting statistics of the point density for four different land cover classes (bare fields, urban, vegetation and water surfaces) are given in Table 3 .

\begin{tabular}{|l|c|r|r|}
\hline \multicolumn{1}{|c|}{ Class } & $\begin{array}{c}\text { Number } \\
\text { of tiles }\end{array}$ & $\begin{array}{c}\text { Mean } \\
\left(\mathrm{pts} / \mathrm{m}^{2}\right)\end{array}$ & $\begin{array}{c}\text { Std. dev. } \\
\left(\mathrm{pts} / \mathrm{m}^{2}\right)\end{array}$ \\
\hline Bare fields & 615,775 & 51.2 & 12.7 \\
Urban & 626,321 & 52.7 & 18.8 \\
Vegetation & 411,953 & 57.3 & 28.5 \\
Water & 673,386 & 10.1 & 7.2 \\
\hline
\end{tabular}

Table 3. Point density by class.

For all classes except water (see Section 3.6), the mean observed density is between 50 and $60 \mathrm{pts} / \mathrm{m}^{2}$, higher than simulated, although the large standard deviations show some significant variation in this and is probably symptomatic of the changing point density across the swath. The histograms in Figure 12, whilst tending towards normal distribution shaped curves, also have long tails which again is probably symptomatic of the irregular point density across the swath width. The sample median may therefore provide a more representative statistic than the sample mean for the majority of the swath width. Nevertheless, of course laterally overlapping data will also be prevalent in complete, merged datasets unless resampling occurs to remove the higher point sampling density at the periphery of the swath that arises from such a scan pattern. 

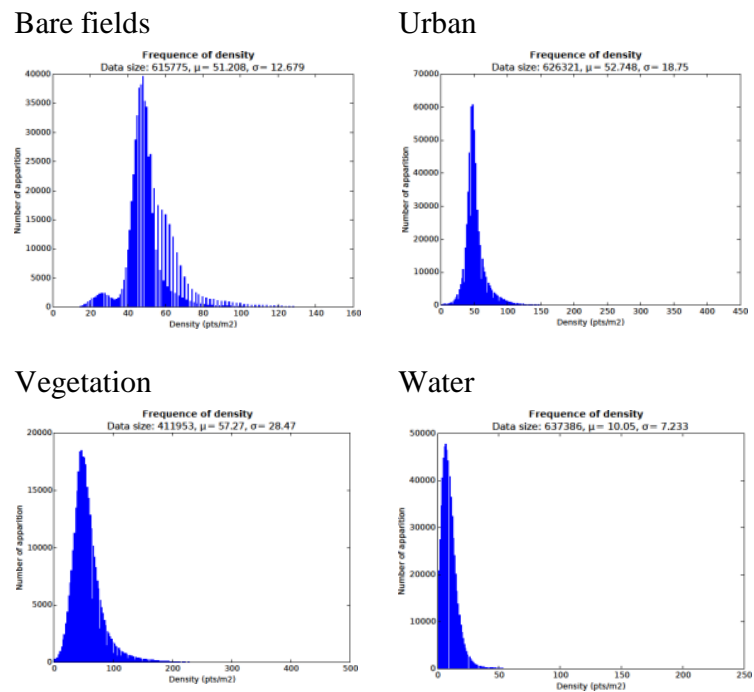

Figure 12. Histograms of point distribution by class (note, to aid interpretation, histograms are displayed with differing scales).

Were the distribution of points regular, the distance between neighbouring points would be equal to $1 / \sqrt{d}$, where $d$ is the measured point density. Thus, for a density of $55 \mathrm{pts} / \mathrm{m}^{2}$, the point spacing would be c. $15 \mathrm{~cm}$ in plan. Although obviously also dependent on point accuracy, such a point spacing may be insufficient for NMCAs to achieve required topographic mapping specifications, but it should be considered that (a) lateral overlaps will potentially greatly increase the point density (Figure 13), and (b) flight parameters could be altered to achieve higher point densities per swath. For instance, the flying height and speed of the Easton datasets were $3750 \mathrm{~m}$ and $370 \mathrm{~km} / \mathrm{h}$, respectively. Decreasing either value would improve the point spacing and, by logical implication, the achievable planimetric precision and accuracy of derived data.

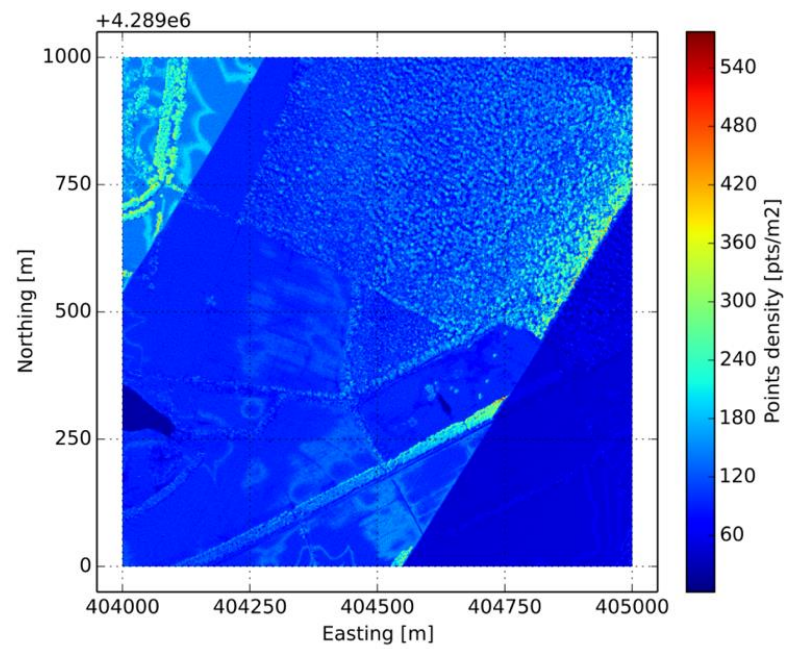

Figure 13. Sample Easton $1 \times 1 \mathrm{~km}$ tile, showing point density in single (bottom right), dual (middle) and triple (top left) lateral overlapping flight lines.

\subsection{Point height precision}

The height precision of the point data over various land cover types was assessed based on a local plane fitting exercise. As previously explained, each of the eight sampled $1 \times 1 \mathrm{~km}$ tiles was subdivided into $1 \mathrm{x} 1 \mathrm{~m}$ sub-tiles, with each point in the cloud belonging to one sub-tile. If all the points contained in a $1 \times 1 \mathrm{~m}$ sub-tile are considered to form part of a local plane, then the deviation of individual points from the best fitting plane can be considered to provide an indication of local precision. The local plane equation for each sub-tile was therefore calculated for different classes and the behaviour of local points on that plane investigated via the standard deviation of the fit. I.e. by estimating the orthogonal distance between the points and the computed plane, the local precision of points was calculated using the standard deviation of the distances (Table 4). The deviations of classified features likely to contain significant discontinuities, such as steep roofs, are not reported.

\begin{tabular}{|l|r|c|r|}
\hline Class & \multicolumn{1}{|c|}{$\begin{array}{c}\text { Number } \\
\text { of pts }\end{array}$} & $\begin{array}{c}\text { Mean } \\
(\mathrm{m})\end{array}$ & $\begin{array}{c}\text { Std. } \\
\text { dev. }(\mathrm{m})\end{array}$ \\
\hline Water & 56,281 & 0.019 & 0.024 \\
Bare fields & 248,077 & 0.025 & 0.008 \\
Ground beneath vegetation & 18,700 & 0.029 & 0.009 \\
Flat roofs & 9,106 & 0.022 & 0.017 \\
Urban ground & 35,151 & 0.026 & 0.008 \\
Roads / car parks & 12,330 & 0.023 & 0.007 \\
\hline
\end{tabular}

Table 4 . Height point precision by class.

The mean standard deviation of the local plane fitting exercise for all classes is in the range 2 to $3 \mathrm{~cm}$. Moreover, the standard deviation of the mean for the fitting exercise is sub-cm except for flat roofs (most probably explained by micro roof topography such as chimneys, antennae and dormer windows) and water surfaces (explained by variable penetration into water bodies). As can be seen by the distributions in Figure 14, offsets approximate to normal distributions in most instances.
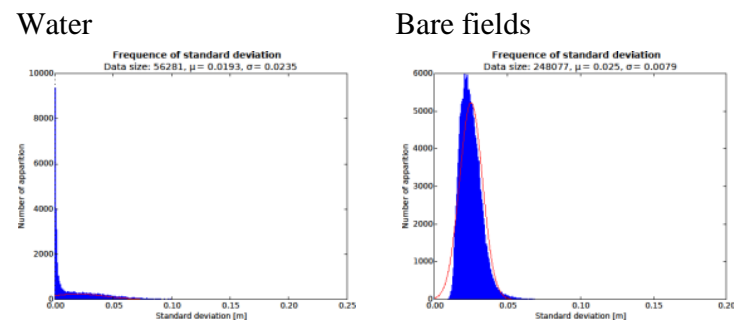

Ground beneath vegetation

Flat roofs
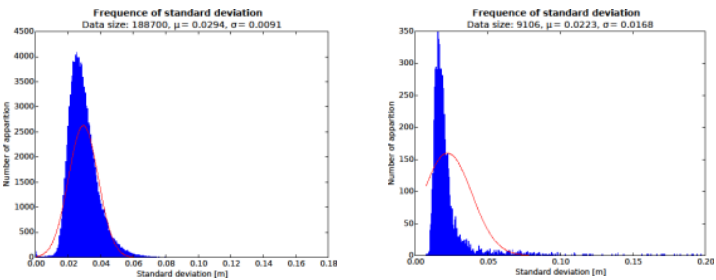

Urban ground

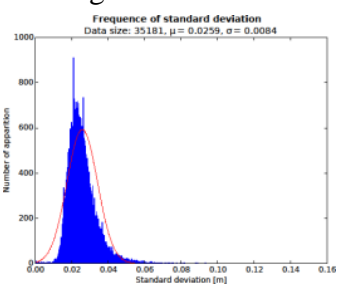

Roads / car parks

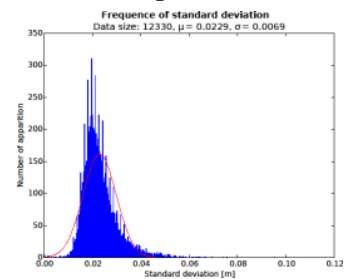

Figure 14. Histograms of height precision by class (note, to aid interpretation, histograms are displayed with differing scales). 


\subsection{Vegetation penetration}

The potential to penetrate vegetation is regarded by NMCAs as one of the most important characteristics of airborne laser scanning for topographic mapping. Figure 15 shows a crosssection taken through an example area of woodland. It is clear from analysis that in some areas of dense vegetation, the Leica SPL100 does not penetrate down to ground level, though further investigations are needed to examine this characteristic.

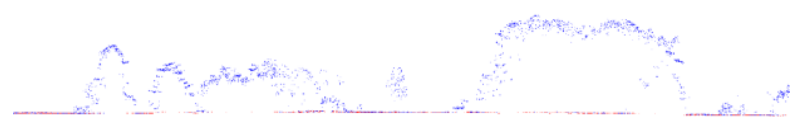

Figure 15. Example of Leica SPL100 vegetation penetration.

\subsection{Bathymetric potential}

Although not promoted as such by the manufacturer, the offnadir $532 \mathrm{~nm}$ (green) wavelength laser that is utilised in the Leica SPL100 inherently displays natural bathymetric potential (Mandlburger and Jutzi, 2018). Investigation therefore also related to the capability of the sensor for penetrating shallow water bodies. The method to determine the capacity for water penetration was to measure differences between the maximum and minimum elevations over areas classified as water. The observations provide an order of magnitude of the penetration into a water body (note, measurements cannot be considered exact since refraction coefficients etc. were not applied).

The left hand histogram in Figure 16 indicates a maximum depth measurement of c. $5 \mathrm{~m}$. Two apparent peaks appear in the histogram: the first with a mean value of c. $1 \mathrm{~m}$ and the second, less pronounced, with values ranging from 2 to $3 \mathrm{~m}$. As the laser is unlikely to reach the bottom in the centre of a water body, the right hand histogram in Figure 16 displays the values only for returns recorded in shallow water close to shore. Two distinct peaks are now observed, this time at c. $1 \mathrm{~m}$ and $2.75 \mathrm{~m}$. The first peak (c. $1 \mathrm{~m}$ ) includes returns received from the water body where the bed is beyond the range of the laser, in addition to any returns received from the bed. The second peak (c. $2.75 \mathrm{~m}$ ) would appear to relate to returns close to the shore where the water is shallow and clear enough for the rays to reach the bed. (a)

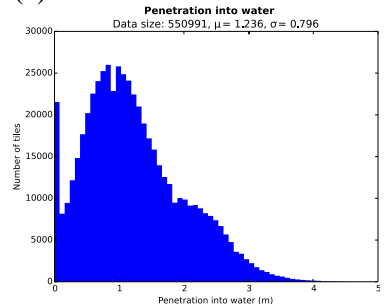

(b)

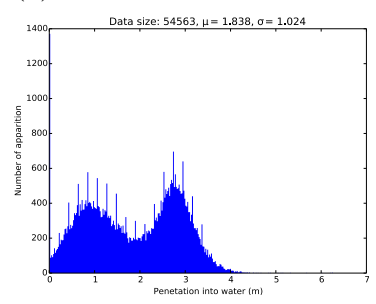

Figure 16. Histograms of water penetration in: (a) all water bodies, and (b) near shore areas only (note, to aid interpretation, histograms are displayed with differing scales).

Thus, it can be concluded that the average (uncorrected) depth penetration in this dataset is equal to c. $2.75 \mathrm{~m}$. Assuming a refractive index for water of 1.333 , this would equate to a corrected maximum depth measurement of c. $2.0 \mathrm{~m}$. Of course, it is important to note that no formal conclusion as to water penetration can be reached without considering prevalent water properties such as turbidity and composition, etc. which will greatly influence the maximum penetration depth. Moreover, it should be recognised that Hexagon categorically state that the sensor is not in any way designed or tuned for bathymetry. Nevertheless, the Leica SPL100 demonstrates considerable potential as a shallow water mapping tool.

\subsection{SP lidar assessment summary}

Preliminary independent analysis of this Leica SPL100 dataset has indicated performance consistent with the manufacturer's claims. SP lidar therefore shows considerable promise for territorial mapping. However, it should be noted that conclusions are drawn on the basis of (a) analysis of a dataset that is now over two years old; (b) unknown pre-processing having been performed; (c) absence of ground truth and alternative comparable sensor data in an unknown and unvisited area.

\section{RECENT AND ONGOING RESEARCH ACTIVITY}

\subsection{EuroSDR Workshop}

A EuroSDR workshop on SP and GM lidar was held at the Institut Cartogràfic i Geològic de Catalunya (ICGC) in Barcelona, Spain, on 6th March 2019. The one-day workshop brought together 65 researchers, developers and practitioners from the global mapping sector to discuss this new airborne laser scanning technology and the results achieved to date. 15 presentations were made, the majority of which can be downloaded from the EuroSDR webpage (EuroSDR, 2019b). The various presented investigations revealed a potential, but somewhat cautious, level of enthusiasm towards the adoption of SP/GM lidar technologies. The lack of available public datasets for critical evaluation, together with the heavy reliance on preprocessing being performed by the data provider, were two of the reasons cited for the cautious approach.

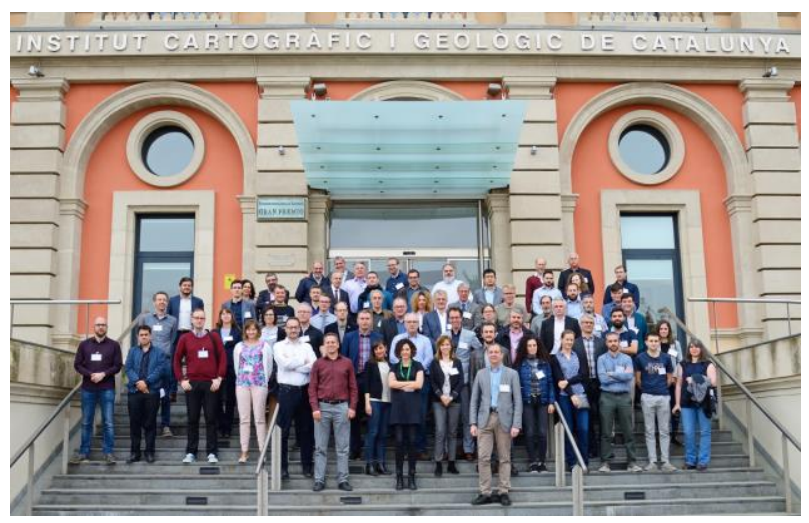

Figure 17. Attendees at the EuroSDR Workshop on SP/GM lidar, Barcelona, Spain on $6^{\text {th }}$ March 2019.

\subsection{EuroSDR Benchmark}

Following feedback on the need for more detailed analysis and understanding into the capabilities of SP and GM lidar, and in conjunction with the promising analysis conducted and reported, a single photon lidar benchmarking project was recently launched by Commission 1. The benchmark was discussed by delegates at the Barcelona workshop and will run for two years and aims to collect different datasets with the support of commercial providers in order to perform detailed investigations and analyses. ISPRS researchers will be welcome 
to participate and are encouraged to contact the authors to register their interest.

\section{CONCLUDING REMARKS}

This paper has reported the aims and progress achieved to date in a EuroSDR initiative to investigate the potential of SP and GM lidar. The mapping community's perspective on the technology has been evaluated through the distribution of an on-line questionnaire, the results of which were reported in Section 2. The outcome revealed some considerable, if cautious, enthusiasm for what the new technology brings to users, with a focus on NMCAs. A preliminary investigation into the performance of a Leica SPL100 sensor has also been undertaken, with results reported in Section 3. Albeit performed with a less than optimal dataset, the preliminary findings endorse the enthusiasm felt for this new technology and its potential future adoption in territorial mapping. Further detailed investigations with various datasets are necessary, as also pointed out in the recent EuroSDR workshop and in the proposed benchmarking activity.

\section{ACKNOWLEDGEMENTS}

The authors would like to thank EuroSDR for supporting this Commission 1 initiative on SP and GM lidar technologies. Hexagon Geosystems is gratefully acknowledged for providing Leica SPL100 data in support of the preliminary investigation and for generous sponsorship of the EuroSDR workshop held in Barcelona, Spain in March 2019. The investigative research reported in Section 3 made use of equipment funded by UKCRIC - UK Collaboratorium for Research in Infrastructure \& Cities: Newcastle Laboratories (EPSRC award EP/R010102/1).

\section{REFERENCES}

Degnan, J. J., 2016. Scanning, multibeam, single photon lidars for rapid, large scale, high resolution, topographic and bathymetric mapping. Remote Sensing, 8, 958. doi:10.3390/rs8110958.

EuroSDR (2019a). EuroSDR. http://www.eurosdr.net/

$(28$ March 2019).

EuroSDR (2019b). EuroSDR one-day workshop on single photon and Geiger-mode lidar. http://www.eurosdr.net/workshops/eurosdr-one-day-workshopsingle-photon-and-geiger-mode-lidar (28 March 2019).

Harris Geospatial Solutions, 2019. Geiger-mode LiDAR. https://www.harris.com/solution/geiger-mode-lidar (28 March 2019).

Hexagon, 2019. Leica SPL100 Single photon LiDAR sensor. https://leica-geosystems.com/en-GB/products/airbornesystems/topographic-lidar-sensors/leica-spl100 (28 March 2019).

Mandlburger, G., Jutzi, B., 2018. Feasibility investigation on single photon LiDAR based water surface mapping. ISPRS Ann. Photogramm. Remote Sens. Spatial Inf. Sci., IV-1, 109-116.

Mandlburger, G., Lehner, H., 2019. Single Photon LiDAR Grundlagen und erste Evaluierungsergebnisse.
Dreiländertagung der DGPF, der OVG und der SGPF in Wien, Österreich - Publikationen der DGPF, Band 28, 443-457.

Stoker, J. M., Abdullah, Q. A., Nayegandhi, A., Winehouse, J., 2016. Evaluation of single photon and Geiger mode lidar for the 3D Elevation Program. Remote Sensing, 8, 767. doi:10.3390/rs8090767.

Wästlund, A, Holmgren, J., Lindberg, E., Olsson, H., 2018. Forest variable estimation using a high altitude single photon Lidar System. Remote Sensing, 10, 1442. doi:10.3390/rs10091442.

Zappa, F., Tisa, S., Tosi, A., Cova, S. 2007. Principles and features of single-photon avalanche diode arrays. Sensors and Actuators A: Physical, 140(1), 103-112. 